\title{
Reply for the Letter to the Editor for the Manuscript "Sleeve Gastrectomy and Roux-y-Gastric Bypass Lead to Comparable Changes in Body Composition After Adjustment for Initial Body Mass Index"
}

Mirko Otto ${ }^{1}$

Published online: 7 June 2016

(C) Springer Science+Business Media New York 2016

I refer to your letter dated 10 March 2016. I am herewith submitting our Reply.

Thanks a lot for your helpful comments regarding our paper.

Body fat index is certainly a good parameter. However, our intention was to demonstrate the problems using the frequently used excess weight loss as target in bariatric surgery. If you want to use a very simple parameter like excess weight loss, total weight loss is superior.

Of course, visceral and subcutaneous fat can be distinguished using magnetic resonance imaging (MRI) or computed tomography (CT). Our group showed that compared to bioelectrical impedance analysis to measure changes in body composition after bariatric procedures, a single-layer MRI was superior in determining visceral fat (1); however, the applicability of CT or MRI to the routine follow-up program after bariatric surgery is surely limited.

Long-term studies examining the differences between gastric bypass and sleeve gastrectomy are definitely necessary, and visceral fat is particularly of interest.
This study compares body compositions which have been acquired through bioelectrical impedance analysis, and we did not aim to make a statement regarding specific fat distribution.

\section{Compliance with Ethical Standards}

Conflict of Interest The authors declare that they have no competing interests.

Ethical Approval Approval from the local institutional review board (IRB) was obtained and is in agreement with the Helsinki declaration.

Statement of Informed Consent Informed consent was obtained from all individual participants included in the study.

\section{Reference}

1. Otto M, Färber J, Haneder S, et al. Postoperative changes in body composition - comparison of bioelectrical impedance analysis and magnetic resonance imaging in bariatric patients. Obes Surg. 2015;25(2):302-9.
Mirko Otto

mirko.otto@umm.de

1 UMM, University Medical Center Mannheim, Department of Surgery, Heidelberg University, Theodor-Kutzer-Ufer 1-3, 68135 Mannheim, Germany 